\title{
Quasi-static resonances in the visible spectrum from all-dielectric intermediate band semiconductor nanocrystals
}

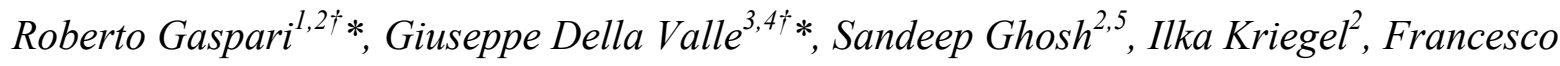
Scotognella ${ }^{3}$, Andrea Cavalli, Liberato Manna ${ }^{2}$

${ }^{1}$ CompuNet, and ${ }^{2}$ Department of Nanochemistry, Istituto Italiano di Tecnologia (IIT), via Morego 30, 16163 Genova, Italy

${ }^{3}$ Dipartimento di Fisica, Politecnico di Milano and ${ }^{4}$ Istituto di Fotonica e Nanotecnologie, Consiglio Nazionale delle Ricerche, Piazza Leonardo da Vinci 32, 20133 Milano, Italy

${ }^{5}$ McKetta Department of Chemical Engineering, The University of Texas at Austin, Austin, Texas 78712-1589, United States

${ }^{\dagger}$ These authors contributed equally to this work.

E-mail: roby.gaspari@gmail.com; giuseppe.dellavalle@polimi.it 


\begin{abstract}
The quest for materials with metal-like properties as alternatives to noble metals is an intense area of research that is set to lead to dramatic improvements in technologies based on plasmonics. Here, we present intermediate band (IB) semiconductor nanocrystals (NCs) as a class of all-dielectric nanomaterials providing quasi-static optical resonances. We show that IB NCs can display a negative permittivity in a broad range of visible wavelengths, enabling a metal-like optical response despite the absence of free carriers in the NC ground state. Using a combination of spectroscopy measurements and ab-initio calculations, we hereby provide a theoretical model for both the linear and nonlinear optical properties of chalcopyrite $\mathrm{CuFeS}_{2}$ NCs, as a case study of IB semiconductor nanomaterials. Our results rationalize the high performance of IB nanomaterials as photothermal agents, and suggest the use of IB semiconductors as alternatives to noble metals for technologies based on plasmonic materials.
\end{abstract}

Keywords: Intermediate-band semiconductors, Quasi-static resonances, All-dielectric nanomaterials, Nonlinear optics, Ultrafast spectroscopy 
Plasmonic phenomena occurring in the visible spectrum are of particular interest, as they exploit the abundancy of sunlight for a wide range of applications, some examples of which are solar harvesting, photo-catalysis and light-to-heat conversion (for an overview, see Refs. 1-2 and references therein). At the nanoscale, visible light excites localized surface plasmon resonances (LSPRs) in nanocrystals (NCs) made of noble metals, such as gold and silver. However, intensive research is now being devoted to the development of alternative nanocrystalline materials ${ }^{3,4}$ with metal-like properties that could outperform noble metalsin aspects regarding absorption loss, ${ }^{5}$ compatibility with silicon manufacturing technologies and integration in metamaterial components. ${ }^{6}$ All-dielectric materials, i.e. materials with no free charges in their ground state, have so far received little attention as possible substitutes for noble metals. One route to obtain large optical responses of all-dielectric NCs in the ultraviolet(UV)/visible regions relies on the excitation of Mie resonances. ${ }^{7}$ However, Mie resonances feature a very different mechanism of excitation to that of LSPRs, as they are based on a phase retardation effect inside the $\mathrm{NC}$ and are not localized. Moreover, they typically require NCs on the length scale of hundreds of nanometers, while LSPRs absorption can occur in much smaller NCs with a typical length of $10 \mathrm{~nm}$ or less. Small all-dielectric NCs can in principle display resonances similar to those exhibited by their noble metal counterparts, since the quasi-static equation for the absorption cross section $\sigma$ of a small nano-object is generally valid for both metallic and non-conducting NCs. This equation reads as: ${ }^{8}$

$$
\sigma=\zeta \frac{\varepsilon_{m} \varepsilon_{i}}{\left(\varepsilon_{r}+2 \varepsilon_{m}\right)^{2}+\varepsilon_{i}^{2}}
$$

In the above equation, $\varepsilon=\varepsilon_{r}+i \varepsilon_{i}$ and $\varepsilon_{m}$ are, respectively, the complex permittivity of the $\mathrm{NC}$ material and that of the medium in which the $\mathrm{NC}$ is dispersed, while $\zeta=24 \pi^{2} R^{3} \sqrt{\varepsilon_{m}} / \lambda$ is a coefficient that depends on the NC radius $R$ and on the excitation wavelength $\lambda$. The quasi-static enhancement of the absorption peak occurs when the denominator in Eq. (1) 
approaches zero, that is when $\varepsilon_{r}=-2 \varepsilon_{m}$ and $\varepsilon_{i} \cong 0$. These relationships represent the socalled Fröhlich condition (FC), and imply a negative real permittivity of the NC material. Negative permittivities typically occur in metals because of the presence of free carriers, allowing for quasi-static plasmonic resonances in the UV-vis range. Similarly to metals, doped semiconductors can also exhibit plasmonic resonances but typically operate in the mid infrared (see e.g. Ref. 9 and references therein), with very few examples (like chalcogenide semiconductors) where the plasmonic resonance can be tuned to the near infrared thanks to heavy doping (including self-doping) (see Ref. 10 for an overview). However, ground state free carriers are absent in all-dielectric materials and negative permittivities can only be caused by interband transitions, typically enabling a FC resonance only in the deep UV. ${ }^{11}$ As an example, collective resonances with energies between 16 and $18 \mathrm{eV}$ which obey the FC have been reported for spherical silicon NCs with radii ranging from 3 to $30 \mathrm{~nm}$. Strategies to obtain visible light, quasi-static resonances in all-dielectric materials are scarce. ${ }^{12}$ Examples are provided by dye-based materials assembled in thin films. ${ }^{13,14}$ Similarly, interband transitions in bulk $\mathrm{Bi}^{15}$ or in the $\mathrm{Bi}_{1.5} \mathrm{Sb}_{0.5} \mathrm{Te}_{1.8} \mathrm{Se}_{1.2}$ topological insulator ${ }^{16}$ generate an extended region of negative permittivities in the UV-vis spectral range, without resorting to the excitation of free carriers.

Here, we propose another route to achieve negative real permittivity in all-dielectric materials and demonstrate its applicability as a tool to excite visible light quasi-static resonances in colloidal NCs. The theoretical foundations of our work are outlined as follows. The dielectric properties of a typical semiconductor in the visible spectrum are dominated by direct optical transitions from the valence band (VB) to the conduction band (CB) (Figure 1a). Each optical transition provides a Lorentz-like contribution to the permittivity, the sum of all the transitions typically causing an extended frequency band of optical absorption (Figure 1a). This can enable negative permittivity and eventually the onset of an FC resonance at high excitation energies. However, an instance can occur in which the CB exhibits a region of very 
low or even zero density of states (DOS): this is the typical scenario for an intermediate band (IB) semiconductor. The second gap suppresses high-energy transitions, and can potentially lead to a dramatic reduction of the FC resonance energy (Figure 1b).

We tested this hypothesis using a model system resembling a prototypical IB semiconductor with a large VB-CB gap and a narrow IB band (see the Supplementary Information for parameter values) under the following simplifying assumptions: (i) VB, IB and CB have uniform DOS (Figure 1c); and (ii) each transition from the VB to the IB or to the $\mathrm{CB}$ contributes to the total permittivity as a single oscillator. Figure 1c shows how the position of the IB (and thus the VB-IB gap) affects the permittivity. The reduction of the VBIB gap dramatically red-shifts the FC resonance energy. On the other hand, increasing the VB-IB gap beyond a threshold value leads to the suppression of the FC resonance mechanism due to the disappearance of the real negative permittivity.
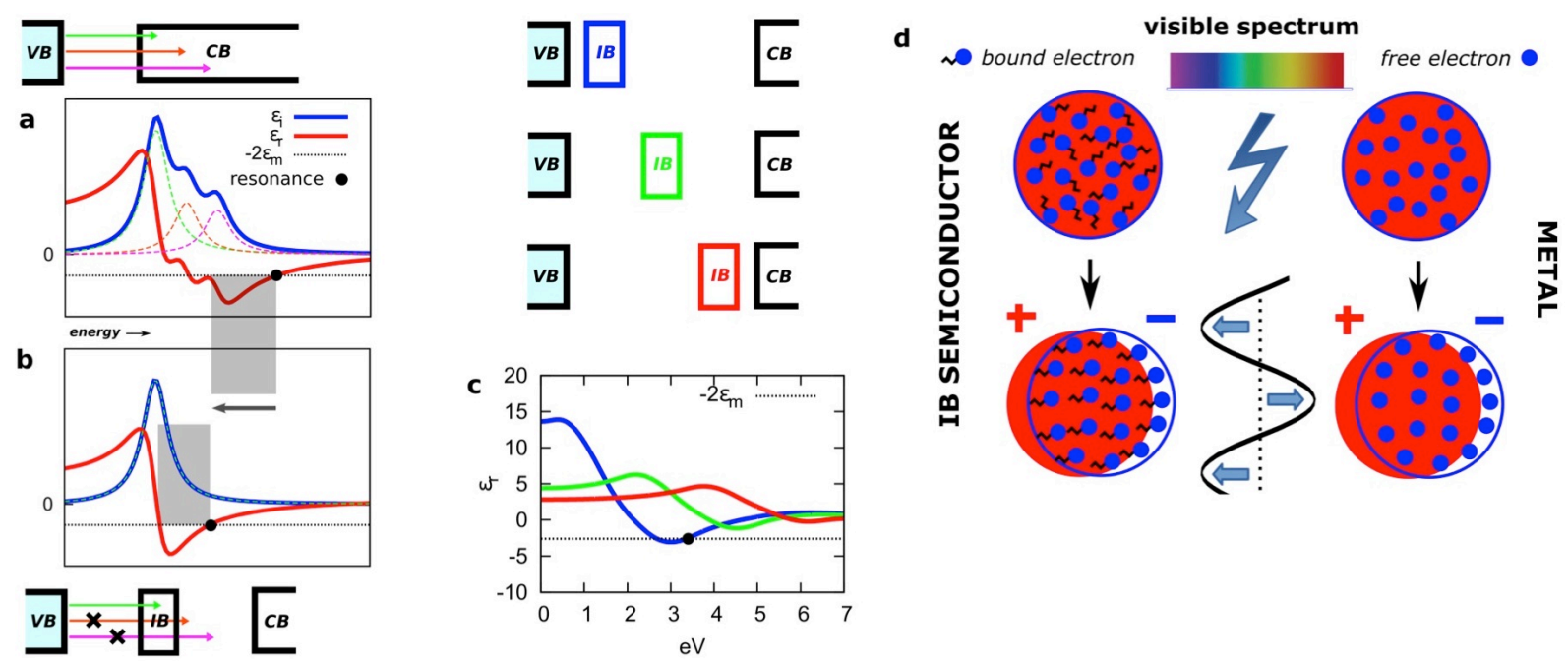

Figure 1. Mechanism of quasi-static resonance in IB semiconductors. (a) Dielectric properties of a conventional semiconductor, exemplified in terms of a few oscillators. The absorption bands of the different oscillators overlap, pushing the resonance to high energies. (b) By setting part of the density of states in the CB to zero, an IB semiconductor is formed. With respect to the non-IB system of (a), this leads to the suppression of some oscillators and to a sharper $\varepsilon_{i}$. By Kramers-Kronig relationships, this results in a strong decrease of the resonance energy. (c) Permittivity curves predicted by the uniform DOS model. Calculation details are reported in SI. By shifting the VB-IB gap from small (blue) to average (green) and large (red) values, the region of negative permittivity increases in energy and finally disappears. (d) The analogy between the quasi-static resonance in IB all-dielectric NCs and metal NCs is pictorially described. 
In the example displayed in Figure 1c, a small spherical NC (with a radius of a few nm) placed in a low permittivity medium would resonate at the edge of the visible region of the spectrum. These considerations encouraged us to further examine IB materials, in order to ascertain the capability of all-dielectric IB NCs to provide quasi-static resonances in the visible (Figure 1d). So far, IB materials have been reported in the context of photovoltaics, with the aim of overcoming the Shockley-Queisser limit for cell efficiency. ${ }^{17}$ Strategies to introduce extra bands in the VB-CB gap of a semiconductor include the use of highly mismatched alloys, quantum confinement and dilute magnetic transition metal doping. ${ }^{18}$ Our focus fell on $\mathrm{CuFeS}_{2}$ (chalcopyrite), which is an abundant mineral and natively displays an IB derived from iron $3 d$-orbitals. ${ }^{19}$ In a previous study, ${ }^{20}$ we investigated the photothermal conversion efficiency of small-sized $\mathrm{CuFeS}_{2} \mathrm{NCs}$ and their potential applications in hyperthermia. Our results showed that ultrasmall $\mathrm{CuFeS}_{2} \mathrm{NCs}$ with an effective radius of about $3.5 \mathrm{~nm}$ (i.e. excluding the organic ligand shell) are excellent light absorbers in the visible range, with a pronounced absorption peak at around $490 \mathrm{~nm} \cdot{ }^{20,21} \mathrm{In}$ an attempt to rationalize this absorption feature, we conducted a first-principle analysis of the material's electronic structure and dielectric response. In Figure 2a, we report the total DOS of $\mathrm{CuFeS}_{2}$, obtained using a DFT $+\mathrm{U}$ approach. ${ }^{22}$ The DOS is characterized by a rather small VB-IB gap $(\approx 0.6 \mathrm{eV}$ according to our estimate $)$ and a larger IB-CB gap $(\approx 1.2 \mathrm{eV})$, in agreement with numerical calculations reported by other groups (see e.g. Refs. 22, 23). Using the randomphase approximation, we computed the frequency dependent permittivity of the material (Figure 2b). Notably, $\mathrm{CuFeS}_{2}$ displays an extended negative region of real permittivity in the visible range, from $480 \mathrm{~nm}$ to $610 \mathrm{~nm}$, in line with earlier reflectivity experiments (cf. with Fig. 5 in Ref. 24). Therefore, the NC absorption peak at around $490 \mathrm{~nm}$ cannot be ascribed to bulk interband transitions (lying at much lower energy, i.e. around $700 \mathrm{~nm}^{24}$ ), but rather to the onset of a quasi-static absorption resonance when the FC is approached (even though not exactly fulfilled because of the limited value of the negative real permittivity). This is 
ascertained by comparing the measured optical absorption spectrum (dots in Figure 2c) with the theoretical NC cross-section obtained from Eq. (1) and the ab-initio computed permittivity (dashed line in Figure 2c). The computed and experimental spectra are in excellent agreement. This result highlights that purely semiconducting IB NCs can have a plasmon-like behavior in the visible range, i.e. they can behave similarly to NCs made of metals such as gold or silver, even though they do not possess a high density of free carriers (Figure 1d). The sensitivity of the absorption peak to the medium permittivity and, in particular, the red-shift of the absorption resonance of the $\mathrm{NC}$ when increasing the permittivity of the environment (Figure 2d) further reinforce the analogy with metal plasmonic NCs (see SI for details).
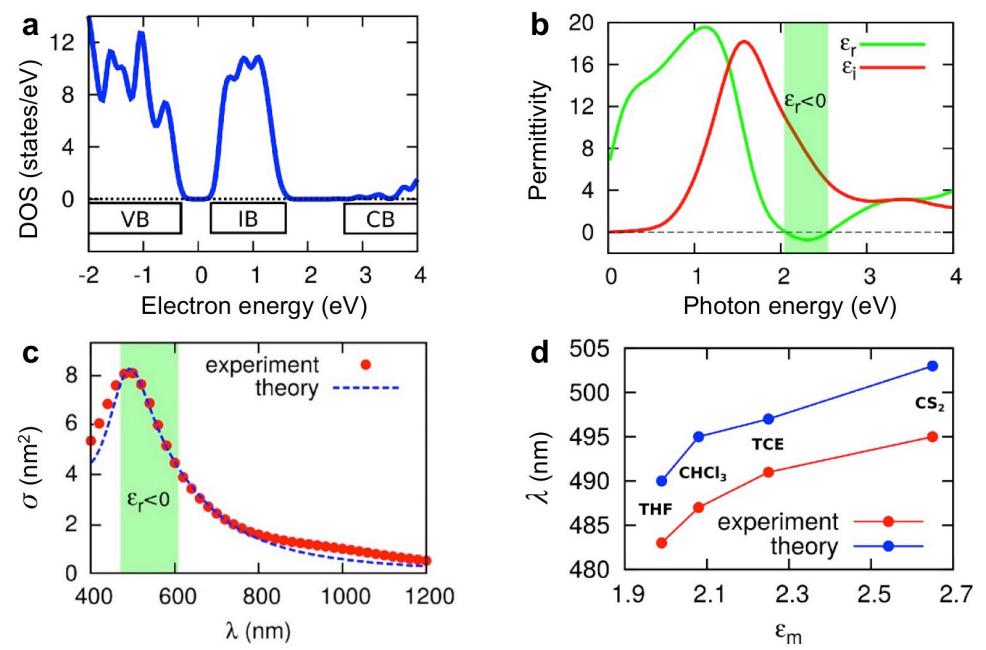

Figure 2. Electronic and optical properties of $\mathrm{CuFeS}_{2}$ NCs. (a) DFT-computed DOS of bulk $\mathrm{CuFeS}_{2}$. (b) Complex permittivity computed at the random phase approximation level. (c) Absorption cross section as obtained by experiments (red dots) or by quasi-static theory and DFT calculations (blue dashed line) for $\mathrm{CuFeS}_{2} \mathrm{NCs}$ dispersed in chloroform. (d) Measured absorption peak position as a function of the environment permittivity. In the present case, the solvents in which the NCs were dispersed are: tetrahydrofuran (THF), chloroform $\left(\mathrm{CHCl}_{3}\right)$, tetrachloroethylene (TCE) and carbon disulfide $\left(\mathrm{CS}_{2}\right)$.

It is worth noting that the figure of merit $\left|\varepsilon_{r} / \varepsilon_{i}\right|(\sim 0.25$ at around $500 \mathrm{~nm})$ is smaller than that typical of noble metal NCs $\left(\sim 1.8\right.$ in the same wavelentgh range $\left.{ }^{11}\right)$ resulting in a reduced amplitude of the optical response of $\mathrm{CuFeS}_{2} \mathrm{NCs}$ at the resonance frequency. Nevertheless, 
from a qualitative standpoint, the features of the excitation in $\mathrm{CuFeS}_{2} \mathrm{NCs}$ are similar to those typical of traditional high-performance plasmonic materials.

The analogy can be further developed beyond the regime of linear optics, i.e. by investigating the nonlinear optical properties of $\mathrm{CuFeS}_{2}$ NCs. Previously ${ }^{20}$, we reported timeresolved spectroscopy measurements to characterize the dynamics of photoexcited electrons and the subsequent modulation of the NC response in the visible range after pump excitation. Here, we demonstrate that these experimental results can be interpreted according to the three-temperature model (3TM) of noble metals ${ }^{25-27}$ and heavily-doped semiconductor ${ }^{28}$ nanostructures. The model describes the dynamics of two populations of out-of-equilibrium carriers, namely: i) non-thermalized carriers generated by the absorption of the pump pulse; and ii) thermalized hot carriers. These two populations are coupled to each other and to the phonons of the NC lattice. In the initial stage of the dynamics, the pulse excitation, described by a time-dependent absorbed power density per unit volume (p.u.v.) $\Phi(t)$, transfers energy to the electronic subsystem via FC-enhanced optical absorption from the VB to the IB. This generates out of equilibrium electrons (in the IB) and holes (in the VB), distributed according to the VB-IB joint density of states, the excess energy density p.u.v. of the carriers being represented by $N(t)$. Over time, these carriers relax either intraband, via carrier-carrier scattering at a rate $a$, or interband, by recombining with rate $b$. The first process is responsible for the formation of a hot gas of thermalized carriers, whose excess energy with respect to the equilibrium temperature $T_{0}$ is detailed by the temperature $T_{E}(t)$. Finally, thermalized hot carriers also couple with phonons and transfer their excess energy to the lattice, whose temperature $T_{L}(t)$ increases with time. The above processes are described by the following system of differential equations: $:^{25-27}$ 


$$
\begin{array}{cl}
\dot{N}(t) & =\Phi(t)-(a+b) N(t) \\
C_{E}\left(T_{E}\right) \dot{T}_{E}(t) & =a N(t)-G\left(T_{E}-T_{L}\right) \\
C_{L} \dot{T}_{L}(t) & =b N(t)+G\left(T_{E}-T_{L}\right)
\end{array}
$$

Here, $G$ is the thermalized electron-phonon coupling coefficient and $C_{E}$ and $C_{L}$ are the heat capacity p.u.v. of the thermalized electrons and lattice, respectively. $C_{E}\left(T_{E}\right) \dot{T}_{E}(t)$ and $C_{L} \dot{T}_{L}(t)$ represent the time derivative of the thermalized electrons' heat $Q_{E}(t)$ and lattice heat $Q_{L}(t)$, (whose expression is given in the SI) due to heating at temperatures $T_{E}(t)$ and $T_{L}(t)$, respectively. The $C_{L}$ coefficient is taken from literature, ${ }^{29}$ whereas $C_{E}$ is retrieved from abinitio calculations (see SI).
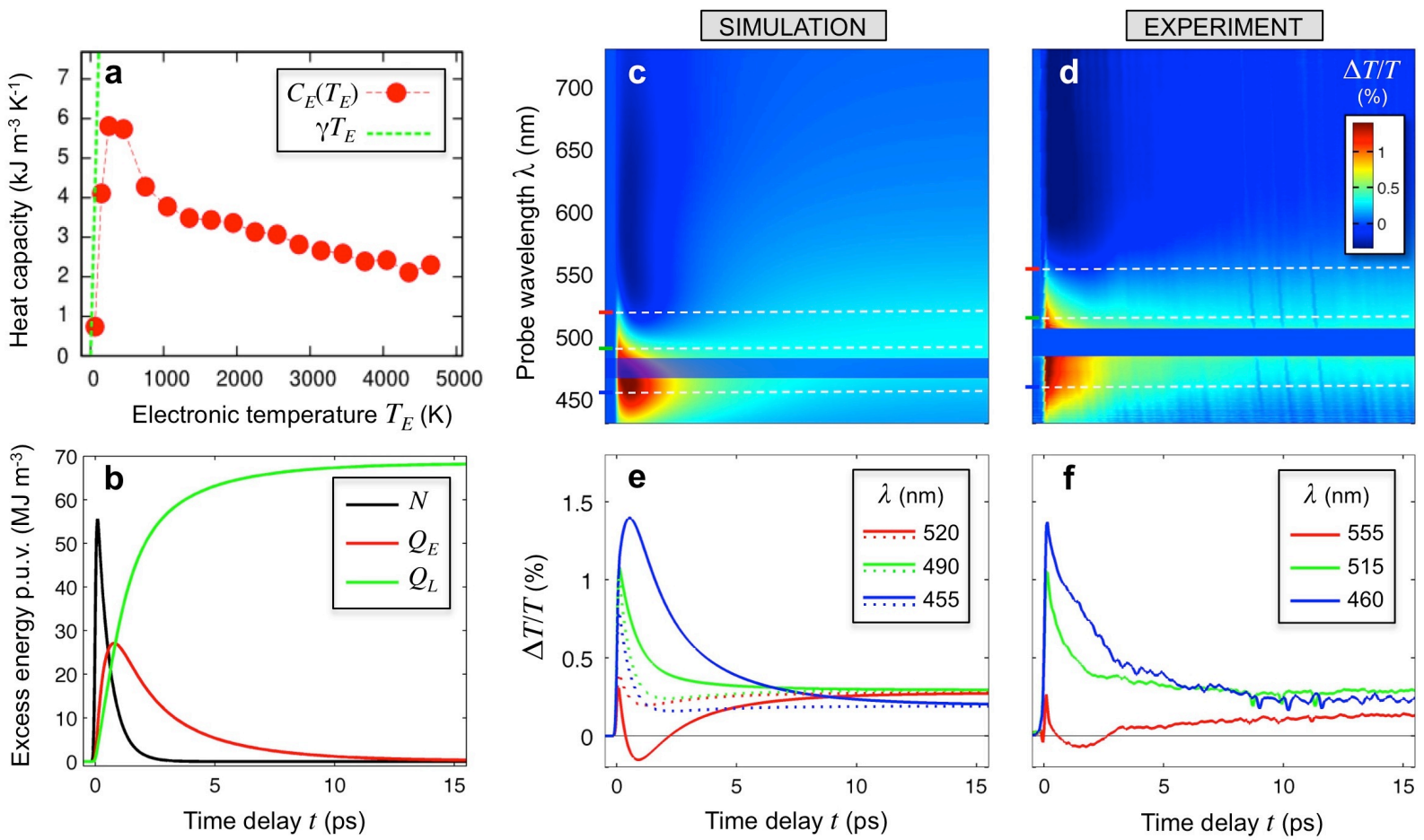

Figure 3. Nonlinear optical response of the $\mathrm{CuFeS}_{2} \mathrm{NCs}$. (a) Heat capacity of the hot electrons as a function of temperature. b) Simulated dynamics of the energy degrees of freedom: excess energy of non-thermalized electrons, $N(t)$, thermalized electrons heat $Q_{E}(t)$, and lattice heat $Q_{L}(t)$ (see SI for details). (c) Simulated and (d) experimental $\Delta T / T(\lambda, t)$ maps. (e)-(f) Temporal cross-sections of the maps in (c) and (d), respectively, at some selected probe wavelengths [corresponding to the dot lines in (c)-(d)]. The dotted lines in panel (e) show the simulated dynamics without considering the contribution from hot electrons.

At low temperature, $C_{E}$ increases linearly with the carriers' temperature $T_{E}$, following the free electrons Sommerfeld relationship $\gamma T_{E}$, but then decreases at higher temperatures (Figure 
3a), thereby displaying a markedly different behavior to that of metallic systems. The negative slope is typical of an IB semiconductor in which the Fermi-Dirac distribution strongly overlaps with the IB-CB gap. In this case, increasing the temperature is inefficient in promoting electrons to higher energy levels because of the vanishing density of states in the IB/CB gap. Consequently, the increase of electronic energy upon heating dramatically reduces in comparison to the case of metals or conventional semiconductors.

The set of Eqs. (2) were then solved numerically, with the experimental pump power density $\Phi(t)$, and $a, b$ and $G$ as fitting parameters. We modeled the optical response of the excited NCs by forming a suitable distribution of non-thermalized and thermalized carriers within the DFT levels of the material's IB and VB corresponding to the typical energy densities retrieved from the 3TM (Figure 3b). From such distributions, we computed the corresponding modulation of the $\mathrm{CuFeS}_{2}$ permittivity function within a perturbative approximation (see SI for further details). Regarding the lattice contribution to the optical response, we assumed a simple thermo-optical mechanism, with a real-valued dispersionless thermo-optic coefficient retrieved from Ref. 30 (for a typical $\mathrm{CuXS}_{2}$ chalcopyrite). The modulated dielectric functions were then used in conjunction with the quasi-static formula of Eq. (1) and the Lambert-Beer law to obtain the simulated $\Delta T / T(\lambda, t)$ map, shown in Figure 3c. Here, $\Delta T(\lambda, t)=T(\lambda, t)-T_{0}(\lambda)$ represents the difference between the transmittance spectrum $T(\lambda, t)$ recorded at a time delay $t$ from the pump pulse arrival and the transmittance spectrum $T_{0}(\lambda)$ of the unperturbed system.

Our simulations were in a good agreement with the experimental map of Figure $3 \mathrm{~d}$ for the following values of the fitting parameters: $a=1.33 p s^{-1} \approx 0.15 a_{A u}, b=0.4 a \approx 1.3 b_{A u}$ and $G=1.8 \cdot 10^{15} \mathrm{~W} \mathrm{~m}^{-3} K^{-1} \approx 0.08 G_{A u}$, in which $a_{A u}, b_{A u}$ and $G_{A u}$ represent the values commonly used for the simulation of gold nanostructures. ${ }^{25,26}$ The rather low values found for the $a$ and $G$ coefficients of $\mathrm{CuFeS}_{2}$ are in line with the semiconducting nature of the material, 
and with the reduced number of states available for electronic transitions in the scattering events. Regarding the value of the $b$ coefficient, despite being close to that of gold, it represents a very different mechanism for electron relaxation compared to that of metals, since it details phonon-assisted interband relaxation, i.e. electron-hole non-radiative recombination (the IB-VB gap being indirect) mediated by defect and surface states. ${ }^{31,32}$ In a typical semiconductor, the latter mechanisms dominate over intraband relaxation processes and, in fact, the optical nonlinearity of all-dielectric nanostructures is commonly modeled according to a rate equation system comprising of not three but two degrees of freedom, $N$ and $T_{L}$, i.e. the hot carriers are disregarded. ${ }^{32,33} \mathrm{We}$ found that an attempt to validate such a reduced model in $\mathrm{CuFeS}_{2} \mathrm{NCs}$ dramatically fails. Actually, hot electrons (and thus the intraband relaxation) can eventually dominate the transient optical response in IB nanomaterials, giving rise to an ultrafast sign change in the $\Delta T / T(\lambda, t)$ (red curve in Figure 3f) that cannot be explained by a simplistic model assuming a single population of carriers (cf. red curves in Figure 3e).

In conclusion, $\mathrm{CuFeS}_{2} \mathrm{NCs}$ can be viewed as the prototype of an innovative class of nanomaterials, being all-dielectric despite exhibiting key optical properties of noble-metals. In particular, $\mathrm{CuFeS}_{2} \mathrm{NCs}$ can provide Fröhlich quasi-static resonances in the visible range and giant optical nonlinearity. Most interestingly, the transient optical response of $\mathrm{CuFeS}_{2} \mathrm{NCs}$ results in the ultrafast non-radiative relaxation of the optically excited free carriers, and the subsequent ultrafast heating of the NC lattice, which is in quantitative agreement with the classic three-temperature model of gold and silver. This provides the basis for a consistent explanation of the high photothermal conversion efficiency reported in IB semiconductor nanomaterials, making them very promising for photothermal therapy. ${ }^{20,34,35}$ IB semiconductors do not show divergences in the dielectric response, and are therefore natural epsilon-near-zero materials over a wide spectral region. For this reason, they are possibly better candidates than noble metals for integration in metamaterials. Furthermore, the 
presence of a non-zero band gap facilitates the separation of electrons and holes upon excitation. This feature, coupled with the intensive heating of the NCs upon light absorption could lay the foundation for interesting applications of IB nanomaterials in photocatalysis. Finally, we foresee applications in other fields of nanophotonics, particularly in $\mathrm{THz}$ alloptical modulation and switching of light, thanks to the ultrafast recovery of the electronic ground state. Recent results from amorphous silicon ${ }^{33}$ and $\mathrm{GaAs}^{32}$ semiconductor metasurfaces further support this scenario, with the demonstration of a modulation capability of about $50 \%$ under $0.3 \mathrm{~mJ} / \mathrm{cm}^{2}$ pump fluence. Even though outperforming $\mathrm{CuFeS}_{2} \mathrm{NCs}$ by almost one order of magnitude in terms of modulation efficiency, the retardation-based nature of the Mie resonance exploited in conventional semiconductors prevents further miniaturization of the individual nanoswitch beyond a fraction of the operation wavelength. A $\mathrm{CuFeS}_{2} \mathrm{NC}$ can exceed this limit in view of the quasi-static nature of the FC-like resonance enabled by its intermediate band.

\section{Associated Content}

The Supporting Information is available free of charge on the ACS Publications website.

\section{Authors contributions}

R.G and G.D.V. designed the work and performed the calculations. They contributed equally to this work. S.G. developed the synthesis of $\mathrm{CuFeS}_{2} \mathrm{NCs}$, characterized them and performed the steady state optical absorption measurements. I.K. and F.S. performed the transient absorption measurements on NC solutions. A.C. and L.M. directed the research. All authors wrote the paper and participated in the scientific discussions. 


\section{Acknowledgments}

The authors acknowledge the IIT platform CompuNet and CINECA for the computational resources. G.D.V. acknowledges support by the Italian MIUR through the PRIN 2015 grant no. 2015WTW7J3 and the COST Action MP1302-NanoSpectroscopy. Dr. Mariella Ippolito is

gratefully acknowledged for the technical support. Dr. Emma De Cecco is gratefully acknowledged for proofreading the paper. 


\section{References}

(1) Schuller, J. A.; Barnard, E. S.; Cai, W.; Jun, Y.; White, J. S.; Brongersma, M L. Nat. Mater. 2010, 9, 193-204.

(2) Atwater, H. A.; Polman, A. Nat. Mater. 2010, 9, 205-213.

(3) Kovalenko, M. V.; Manna, L.; Cabot, A.; Hens, Z.; Talapin, D. V.; Kagan, C. R.; Klimov, V. I.; Rogach, A. L.; Reiss, P.; Milliron D. J.; et al. ACS Nano 2015, 9, 10121057.

(4) Naik, G. V.; Shalaev, V. M.; Boltasseva, A. Adv. Mater. 2013, 25, 3264-3294.

(5) Khurgin, J. B.; Sun, G. Appl. Phys. Lett. 2010, 96, 181102.

(6) Boltasseva, A.; Atwater, H. A. Science 2011, 331, 290-291.

(7) Kuznetsov, A. I.; Miroshnichenko, A. E.; Brongersma, M. L.; Kivshar, Y. S.; Luk'yanchuk, B. Science 2016, 354, aag2472.

(8) Bohren, C. F.; Huffman, D. R. Absorption and Scattering of Light by Small Particles. (John Wiley \& Sons, 1983).

(9) Lewi, T.; Iyer, P. P.; Butakov, N. A.; Mikhailovsky, A. A.; Schuller J. A. Nano Lett. 2015, 15, 8188-8193.

(10) Scotognella, F.; Della Valle, G.; Kandada A. R. S.; Zavelani-Rossi M.; Longhi S.; Lanzani G.; Tassone F. Eur. Phys. J. B 2013, 86, 154.

(11) Raether, H. Excitation of Plasmons and Interband Transitions by Electrons. (Springer Berlin Heidelberg, 1980).

(12) Toudert, J.; Serna, R. Opt. Mater. Express 2017, 7, 2299-2325.

(13) Gentile, M. J.; Núñez-Sánchez, S.; Barnes, W. L. Nano Lett. 2014, 14, 2339-2344.

(14) Gu, L.; Livenere, J.; Zhu, G.; Narimanov, E. E.; Noginov, M. A. Appl. Phys. Lett. 2013, 103, 021104 .

(15) Toudert, J.; Serna, R.; Camps, I.; Wojcik, J.; Mascher, P.; Rebollar, E.; Ezquerra, T. A. J. Phys. Chem. C 2017, 121, 3511-3521. 
(16) Ou, J.-Y.; So J.-K.; Adamo, G.; Sulaev, A.; Wang, L.; Zheludev, N. Nat. Commun. 2014, 5, 5139 .

(17) Luque, A.; Marti, A.; Stanley, C. Nat. Photon. 2012, 6, 146-152.

(18) Okada, Y.; Ekins-Daukes, N. J.; Kita, T.; Tamaki, R.; Yoshida, M.; Pusch, A.; Hess, O.; Phillips, C. C.; Farrell, D. J.; Yoshida, K.; et al. Appl. Phys. Rev. 2015, 2, 021302.

(19) Hamajima, T.; Kambara, T.; Gondaira, K. I.; Oguchi, T. Phys. Rev. B 1981, 24, 33493353.

(20) Ghosh, S.; Avellini, T.; Petrelli, A.; Kriegel, I.; Gaspari, R.; Almeida, G.; Bertoni, G.; Cavalli, A.; Scotognella, F.; Pellegrino, T.; Manna, L. Chem. Mater. 2016, 28, 48484858 .

(21) Gabka, G.; Bujak, P.; Ostrowski, A.; Tomaszewski, W.; Lisowski, W.; Sobczak, J. W.; Pron, A.† Inorg. Chem. 2016, 55, 6660-6669.

(22) Himmetoglu, B.; Floris, A.; de Gironcoli, S.; Cococcioni, M. Int. J. Quantum Chem. 2014, 114, 14-49.

(23) Zhou, M.; Gao, X.; Cheng, Y.; Chen, X.; Cai, L. App. Phys. A 2015, 118, 1145-1152.

(24) Oguchi, T.; Sato, K.; Teranishi, T. J. Phys. Soc. Jpn. 1980, 48, 123-128.

(25) Conforti, M.; Della Valle, G. Phys. Rev. B 2012, 85, 245423.

(26) Sun, C. K.; Vallée, F.; Acioli, L. H.; Ippen, E. P.; Fujimoto, J. G. Phys. Rev. B 1994, $50,15337-15348$.

(27) Pluchery, O.; Louis, C. Gold nanoparticles for physics, chemistry and biology. (World Scientific, 2012).

(28) Della Valle, G.; Scotognella, F.; Kandada, A. R. S.; Zavelani-Rossi, M.; Li, H.; Conforti, M.; Longhi, S.; Manna, L.; Lanzani, G.; Tassone, F. J. Phys. Chem. Lett. 2013, 4, 3337-3344.

(29) Robie, R. A.; Wiggins, L. B.; Barton Jr, P. B.; Hemingway, B. S. J. Chem. Therm. 1985, $17,481-488$. 
(30) Bhar, G. C.; Samanta, L. K. Phys. Stat. Sol. (a) 1980, 57, 735-740.

(31) Bhattacharyya, B.; Pandey, A. J. Am. Chem. Soc. 2016, 138, 10207-10213.

(32) Shcherbakov, M. R.; Liu, S.; Zubyuk, V. V.; Vaski, A.; Vabishchevich, P. P.; Keeler, G.; Pertsch, T.; Dolgova, T. V.; Staude, I.; Brener, I.; Fedyanin, A. A. Nat. Commun. 2017, $8,17$.

(33) Shcherbakov, M. R.; Vabishchevich, P. P.; Shorokhov, A. S.; Chong, K. E.; Choi, D.Y.; Staude, I.; Miroshnichenko, A. E.; Neshev, D. N.; Fedyanin, A. A.; Kivshar, Y. S. Nano Lett. 2015, 15, 6985-6990.

(34) Jiang, X.; Jiang, X.; Zhang, S.; Ren, F.; Chen, L.; Zeng, J.; Zhu, M.; Cheng, Z.; Gao, M.; Li. Z. ACS Nano 2017, 11, 5633-5645.

(35) Li, B.; Yuan, F.; He, G.; Han, X.; Wang, X.; Qin, J.; Guo, Z. X.; Lu, X.; Wang, Q.; Parkin, I. P.; Wu, C. Adv. Funct. Mater. 2017, 27, 1606218. 


\section{For Table of Contents Use Only}

\section{Quasi-static resonances in the visible spectrum from all-}

\section{dielectric intermediate band semiconductor nanocrystals}

Roberto Gaspari, Giuseppe Della Valle, Sandeep Ghosh, Ilka Kriegel, Francesco

Scotognella, Andrea Cavalli, Liberato Manna
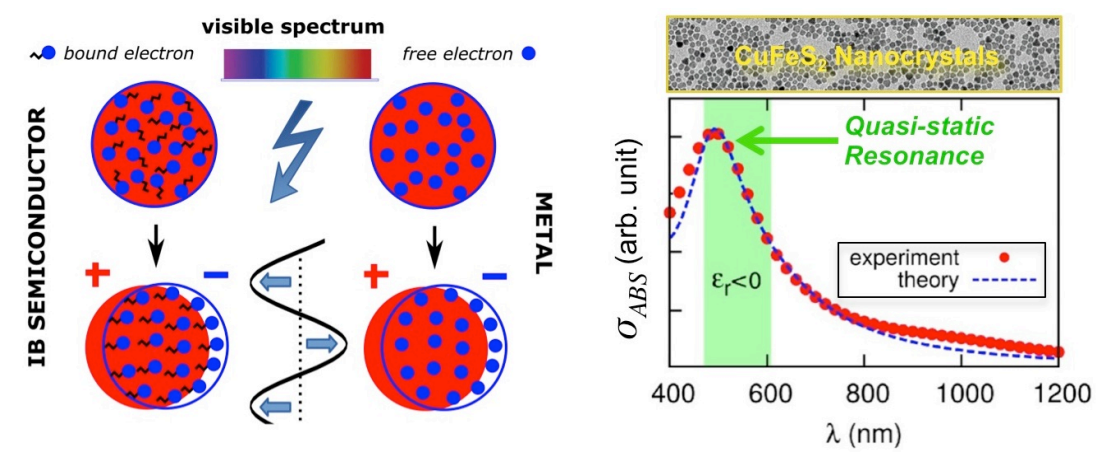

Sketch of the mechanism allowing intermediate-band semiconductor nanocrystals to exhibit quasi-static resonances, in analogy with metallic nanocrystals but without free carriers (left panel). Ab-initio calculations of the linear optical properties of $\mathrm{CuFeS}_{2}$ nanocrystals with few $\mathrm{nm}$ radius, validated on experimental data, confirm the achievement of a Fröhlich-like condition (i.e. absorption resonance in the quasi-static limit enabled by a negative real permittivity) in all-dielectric nanocrystals in the visible range (right panel). 